\title{
Synthesis and Characterisation of Photo-Cross-Linkable Liquid Crystalline Poly(n-[n'-flurobenzoylstyryloxy]alkylmethacrylate)s and Their Fluorescence Lifetime Properties
}

\author{
G. Kumar, ${ }^{1}$ K. Subramanian, ${ }^{1}$ and S. Ganesan ${ }^{2}$ \\ ${ }^{1}$ Department of Chemistry, Anna University, Chennai 600 025, India \\ ${ }^{2}$ Department of Medical Physics, Anna University, Chennai 600 025, India \\ Correspondence should be addressed to K. Subramanian; kathsubramanian@yahoo.com
}

Received 22 June 2012; Accepted 16 January 2013

Academic Editor: Khalique Ahmed

Copyright (c) 2013 G. Kumar et al. This is an open access article distributed under the Creative Commons Attribution License, which permits unrestricted use, distribution, and reproduction in any medium, provided the original work is properly cited.

\begin{abstract}
This paper reports a study on photo-cross-linkable polymer containing pendant chalcone moiety exhibiting liquid crystalline as well as fluorescence lifetime properties in detail. The photoresponsive polymers were prepared, and their structure has been characterized by ${ }^{1} \mathrm{H}-\mathrm{NMR},{ }^{13} \mathrm{C}-\mathrm{NMR}$, and UV-Visible spectroscopy. The photo-cross-linking behavior of polymers has been studied by UV-Visible and fluorescence spectroscopy. UV spectral studies revealed that the polymers follow $2 \pi+2 \pi$ cyclo addition reactions when they undergo photo-cross-linking under the influence of UV-light. Number and weight average molecular weight of the polymers were determined by Gel Permeation Chromatography (GPC) and polydispersity index value near to 1.5. The thermal and thermooxidative stability of the polymers were determined by Thermogravimetric Analysis (TGA). Thermal transitions were studied by DSC, and presence of mesophases was identified at 147 and $126^{\circ} \mathrm{C}$ by hot stage polarized light optical microscopy (HPOM). Fluorescence lifetime measurements using the time-correlated single photon counting (TCSPC) method reveal that the average lifetime values decrease from $5.94 \mathrm{~ns}$ to $5.32 \mathrm{~ns}$ on UV-irradiation were discussed in detail.
\end{abstract}

\section{Introduction}

Liquid crystalline polymers have generated considerable interest in recent years, and the photo-cross-linkable LCPs have driven special attention if they contain both mesogen and photoactive groups in their structure [1-5]. The former incorporates LC properties to the polymer, and the later facilitates cross-linking of the chain under the influence of UV radiation. These classes of polymers are very useful in fabricating anisotropic networks, information storage devices [6, 7] and nonlinear optical devices [8]. Many research articles reported photochemical and liquid crystalline behaviours of these polymers using UV-Visible spectral studies and polarised optical microscopic characterisations. In addition to these studies, fluorescence lifetime measurement has been used as a new instrumental technique to support photoreactive behaviour of photo-cross-linkable liquid crystalline polymers.
Fluorescence lifetime measurements encompass tremendously large fields of science. Since the mid-19th century, nearly every great breakthrough in chemistry and physics has aided the development of fluorescence lifetime techniques, and a growing number of discoveries in biology and medicine owe their existence to fluorescence lifetime. A variety of fluorescence detection methods are available for lifetime measurements but the advent of time-correlated single photon counting (TCSPC) $[9,10]$ has simplified data collection and enhanced quantitative photon counting.

This paper reports newer root for the synthesis of poly(n[ $\mathrm{n}^{\prime}$-flurobenzoylstyryloxy] alkylmethacrylate)s; the photocross-linking and liquid crystalline behaviour of polymers have been well characterised by UV and HPOM studies. We believe that there is no report in recent years on fluorescence lifetime study in combination with UV and liquid crystalline mesophase transition studies about photo-cross-linkable liquid crystalline polymer containing pendant chalcone moiety. 


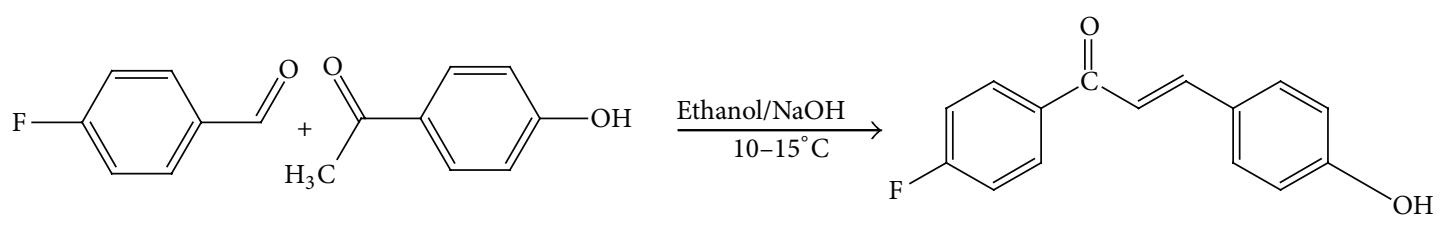

Scheme 1: Synthesis of HPFSK.

This research work may kindle significant scientific work and practical contribution with respect to the development of unique photo-cross-linkable liquid crystalline polymeric materials.

\section{Experimental}

2.1. Materials. 4-Fluorobenzaldehyde and 4-hydroxyacetophe none were purchased from Spectrochem Chemicals. 4-bromobutanols, 6-bromoheaxnol, methacryloyl chloride were purchased from Aldrich Chemicals. Ethanol, tetrahydrofuran, ethylacetate, chloroform, and diethylether were purchased from Merck, and all the solvents were distilled as per standard methods. Thin Layer Chromatography (TLC) technique was carried out on Merck aluminium plates with $0.2 \mathrm{~mm}$ silica gel. Anhydrous sodium sulphate was used to dry all organic extracts. AIBN was recrystallised using 1:1 methanol and chloroform.

2.2. Measurements. The FT-IR spectra of the polymers were recorded on Perkin Elmer FT-IR Spectrometer RXI. The specimen was prepared in the pellet form using $\mathrm{KBr} .{ }^{1} \mathrm{H}$ NMR spectroscopic measurement was recorded with Bruker MSC 300 spectrometer. Thermal stability of polymers was investigated by TGA using NETZSCH STA $409 \mathrm{C} / \mathrm{CD}$. The number average and weight average molecular weight of the polymer were determined by PL-GPC 650. Glass transition temperature of polymer was measured from Differential Scanning Calorimeter (DSC) NETZSCH.DSC.204. The photo-cross-linking studies have been done by Perkin Elmer Lambda 35 UV-Visible Spectrometer. The fluorescence spectrum of the polymer has been recorded in FluroMax 2.0. The texture of the prepared sample was studied by Euromax polarizing microscope equipped with a Linken HFS91 heating stage. The sample was prepared by a small quantity of the material being melted between two thin glass cover slips to get uniform film and anisotropic behavior observed by heating as well as cooling with Toshiba digital camera.

2.2.1. Fluorescence Lifetime Measurements. Lifetime measurements were made using time-correlated single photon counting system (TCSPC, HORIBA JOBIN YUVON IBH, UK) by exciting the sample using $280 \mathrm{~nm}$ Nano-LED (pulse width: <1 ns) and $460 \mathrm{~nm}$ Nano-LED (pulse width: >1 ns), a fast response red sensitive PMT (Hamamatsu Photonics, Japan) detector. The fluorescence emission was collected to 90 degree from the path of the light source. The electrical signal was amplified by a TB-02 pulse amplifier (Horiba) fed to the constant fraction discriminator (CFD, Phillips, The Netherlands). The first detected photon was used as a start signal by a time-to-amplitude converter (TAC), and the excitation pulse triggered the stop signal. The multichannel analyzer (MCA) recorded repetitive start-stop signals from the TAC and generated a histogram of photons as a function of timecalibrated channels (55.7 ps/channel) until the peak signal reached 1000 counts. The instrument response function was obtained using a Rayleigh scatter of Ludox-40 (40 wt.\% suspension in water; Sigma-Aldrich) in a quartz cuvette at $280 \mathrm{~nm}$ excitation and $460 \mathrm{~nm}$ excitation. Decay analysis software (DAS6 v6.0, Horiba) was used to extract the lifetime components. The goodness of fit was judged by the chisquare values, Durbin-Watson parameters, as well as visual observations of fitted line, residuals, and autocorrelation functions.

2.3. Synthesis of Pendant Chalcone (Scheme 1). The pendant chalcone compound HPFSK was synthesized according to the reported literatures $[11,12]$. The obtained products were purified by recrystallisation in ethanol and then identified using ${ }^{1} \mathrm{H},{ }^{13} \mathrm{C}-\mathrm{NMR}$, and FT-IR spectra.

2.4. Hydroxyphenyl-4'-flurostyryl Ketone (HPFSK). In a three-necked flask equipped with a mechanical stirrer and dropping funnel, a solution of $\mathrm{NaOH}(8 \mathrm{~g})$ in distilled water $(40 \mathrm{~mL})$ was added to 4-hydroxyacetophenone $(6.80 \mathrm{~g}, 0.05 \mathrm{~mol})$ in $50 \mathrm{~mL}$ of ethyl alcohol. The reaction was cooled using an ice bath $\left(10-15^{\circ} \mathrm{C}\right)$. A solution of 4fluorobenzaldehyde in $50 \mathrm{~mL}$ of ethyl alcohol was then added dropwise with constant stirring, and the temperature was not allowed to exceed $25^{\circ} \mathrm{C}$. After $12 \mathrm{~h}$, the reaction mixture was neutralized with $2 \mathrm{M} \mathrm{HCl}$ to isolate the product. The yellow coloured solid product was filtered and washed several times with ice-cold water. The crude product was recrystallized from methanol into a yellow crystalline product HPFSK (Scheme 1).

FT-IR (KBr pellet, $\mathrm{cm}^{-1}$ ); 1497 (aromatic C=C); 1590 (olefinic $\mathrm{CH}=\mathrm{CH}) ; 1650$ (keto $\mathrm{C}=\mathrm{O}) ; 3550(\mathrm{Ar}-\mathrm{OH}) .{ }^{1} \mathrm{H}-\mathrm{NMR}$ $\left(\mathrm{CDCl}_{3}, \partial\right.$ in ppm) (Figure 1); 6.8-8 (2d, 8H, Ar-H), 7.5, 7.8 $(2 \mathrm{~d}, 2 \mathrm{H}$ olefinic $-\mathrm{CH}=\mathrm{CH}-)$, and $6.4(\mathrm{~S}, 1 \mathrm{H},-\mathrm{OH})$.

2.5. 4-[4'-Flurobenzoylstyryloxy]butyl Methacrylate (FBSOBMA) (M1) (Scheme 2). In a two-necked round bottom flask, chalcone (HPFSK) ( $3 \mathrm{~g}, 0.0123 \mathrm{~mol})$ was dissolved in $100 \mathrm{~mL}$ of DMF and stirred well, and $\mathrm{K}_{2} \mathrm{CO}_{3}$ $(3.39 \mathrm{~g}, 0.0246 \mathrm{~mol})$ and a pinch of KI were added into the above solution and allowed stiring for 30 minutes in 
<smiles>CC(C)(O)CC(C)(C)Br</smiles><smiles>C=C(C)C(=O)Cl</smiles>

Monomers (M1-M2)

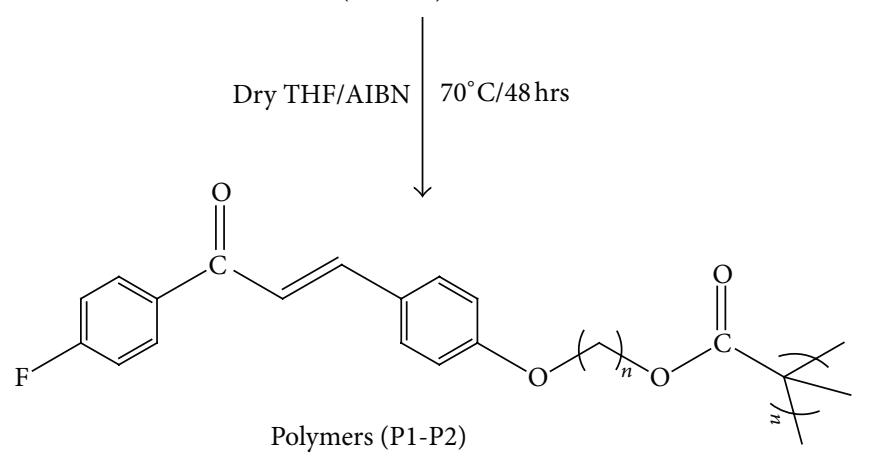

SCHEME 2: Synthesis of monomers and polymers.

an oil bath. The prepared 4-bromobutanol $(1.6 \mathrm{~mL})$ was added dropwise into the above mixture and allowed stirring for $24 \mathrm{~h}$ at $90^{\circ} \mathrm{C}$. The product yellow solid formed was poured into water, filtered, and dried. The crude product 4-hydroxybutyloxystyryl-4'-flurophenyl ketone obtained was recrystallised from ethanol-water mixture $(50: 50)$ (yield $65 \%, 2.5 \mathrm{~g})$.

4-Hydroxybutyloxystyryl-4-flurophenyl ketone (2 g, $6.3 \mathrm{mmol}$ ) and $1.5 \mathrm{~mL}$ of triethylamine were dissolved in ethylmethylketone $(150 \mathrm{~mL})$. The above mixture was cooled between 0 to $-5^{\circ} \mathrm{C}$, and methacryloyl chloride $(2 \mathrm{~mL}$ in $20 \mathrm{~mL}$ of EMK) was added drop wise for an hour with constant stirring and cooling. The reaction mixture was stirred for another 6 hours at room temperature, and the precipitated ammonium salt was filtered off. After drying over anhydrous sodium sulphate, EMK was evaporated using rotary evaporator. The crude monomer product was purified by column chromatography using ethyl acetate/n-hexane $(2: 8 \mathrm{v} / \mathrm{v})$ as eluent. The monomer FBSOBMA (Figure 2) obtained was pale yellow coloured solid (yield 75\%).

${ }^{1} \mathrm{H}$-NMR (Figure 2) $\left(\mathrm{CDCl}_{3}, \partial\right.$ in ppm); 7-8.3 (aromatic protons, $\mathrm{m} 8 \mathrm{H}$ ); 7.5 and 7.9 (olefinic double bond, $2 \mathrm{~d}, 2 \mathrm{H}$ ); $1.3-1.7$ and 3.3-4.3 (spacer ethylenic protons $\mathrm{m}, 8 \mathrm{H}$ ); 5.8 and 6.5 (vinylic protons, d, $2 \mathrm{H}$ ); 2.1 (methyl protons $\mathrm{t}, 3 \mathrm{H}$ ).

\subsection{6-[4'-Flurobenzoylstyryloxy]hexyl Methacrylate} (FBSOHMA) (M2) (Scheme 2). The monomer 6- $\left[4^{\prime}-\right.$ flurobenzoylstyryloxy] hexyl methacrylate (FBSOHMA) (Figure 3) was synthesised followed by analogous procedure discussed in the above monomer synthesis using 6-bromo 


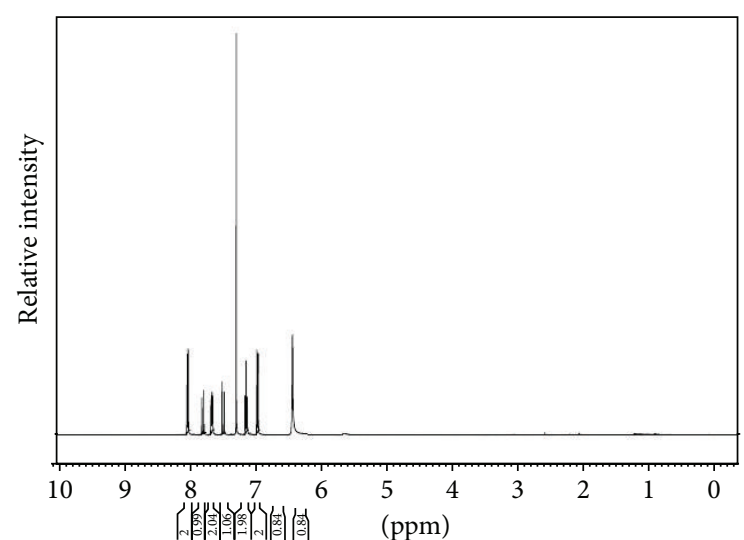

FIGURE 1: ${ }^{1} \mathrm{H}$-NMR spectrum of 4-hydroxyphenyl-4 ${ }^{\prime}$-flurostyryl ketone (HPFSK).

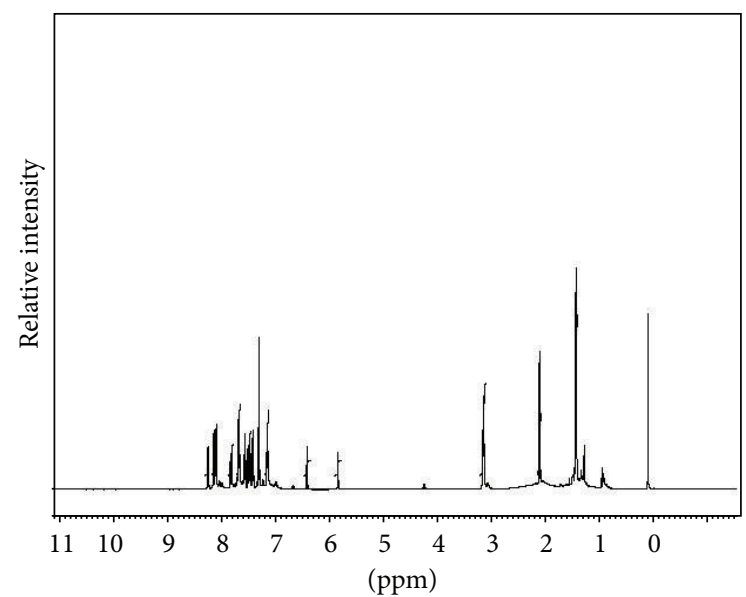

Figure $2:{ }^{1} \mathrm{H}$-NMR spectrum of $\left(4\right.$ - $\left[4^{\prime}\right.$-flurobenzoylstyryloxy $]$ butyl methacrylate) (FBSOBMA) (M1).

hexanol instead of 4-bromobutanol. The monomer obtained was pale yellow coloured solid (yield 67\%).

${ }^{1} \mathrm{H}-\mathrm{NMR}$ (Figure 3) $\left(\mathrm{CDCl}_{3}, \partial\right.$ in ppm); 6.7-7.9 (aromatic protons, $\mathrm{m} 8 \mathrm{H}$ ); 7.5 and 7.9 (olefinic double bond, $2 \mathrm{~d}, 2 \mathrm{H}$ ); 0.9-1.9 (spacer ethylenic protons $\mathrm{m}, 12 \mathrm{H}$ ) 5.8 and 6.3 (vinylic protons, d, 2H); 2 (Methyl protons $\mathrm{t}, 3 \mathrm{H}$ ).

2.7. Synthesis of Polymers (Scheme 2). The polymers poly (4-[4'-flurobenzoylstyryloxy] butyl methacrylate) (P1) and poly(6-[ $4^{\prime}$-flurobenzoylstyryloxy] hexyl methacrylate) (P2) were synthesised by free radical polymerisation. The free radical polymerizations of monomer M1 and M2 were carried out using AIBN as initiator as shown in the schematic representation (Figure 2). The predetermined quantities of monomers were taken with AIBN (5\% weight of monomer) in polymerization tube and dissolved with $20 \mathrm{~mL}$ of dry tetrahydrofuran (THF). The above solutions were degassed by $\mathrm{N}_{2}$ gas atmosphere for $10-15$ minutes. Then, the polymerization tubes were kept at $70^{\circ} \mathrm{C}$ for 48 hours and subsequently then poured into methanol to precipitate the polymer. The polymers obtained were separated by filtration and purified

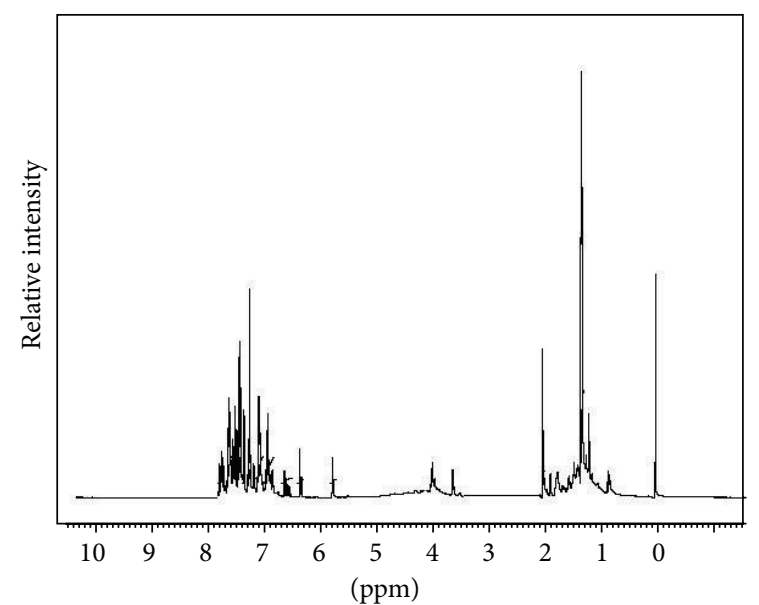

Figure 3: ${ }^{1} \mathrm{H}$-NMR spectrum of (6-[4'-flurobenzoylstyryloxy] hexyl methacrylate) (FBSOHMA) (M2).

by repeated reprecipitation from chloroform into methanol and then dried in vacuum. The yield obtained was $70 \%$.

\section{Photoreactive Measurements}

The photoreactivity of polymers was studied by dissolving the samples in chloroform, irradiated with UV-light at $254 \mathrm{~nm}$ using photoreactor, and kept at a distance of $10 \mathrm{~cm}$ from the light source for different time intervals. After each irradiation period, the UV spectra were recorded using Perkin Elmer scanning spectrometer. The rate of disappearance of double bond in photosensitive group was followed by the expression,

$$
\text { Rate of conversion }(\%)=\frac{\left(A_{o}-A_{t}\right)}{\left(A_{o}-A_{\infty}\right)} \times 100 \text {, }
$$

where $A_{o}, A_{t}$, and $A_{\infty}$ are absorption intensities due to the $>\mathrm{C}=\mathrm{C}<$ group after the irradiation time $t=0, t=T$, and $t=\infty$ (maximum irradiation time), respectively.

\section{Results and Discussions}

4.1. Synthesis and Characterization. The photo-cross-linkable liquid crystalline monomers and polymers were prepared as shown in Scheme 2. The photoreactive chalcone HPFSK was prepared by reacting 4-Fluorobenzaldehyde with 4hydroxyacetophenone in the presence of $\mathrm{NaOH}$ as base. The FT-IR spectrum of HPFSK showed absorption bands at 1497, 1590 , and $3550 \mathrm{~cm}^{-1}$ corresponding to aromatic, olefinic, and alcoholic group, respectively. The ${ }^{1} \mathrm{H}-\mathrm{NMR}$ spectrum (Figure 1) showed resonance signals at $6.8-8,7.5,7.8$, and $6.4 \mathrm{ppm}$ corresponding to aromatic, olefinic, and alcoholic protons, respectively. The monomers were synthesised by reacting the intermediate (formed by O-alkylation of chalcone in DMF in the presence of $\mathrm{K}_{2} \mathrm{CO}_{3}$ ) with methacryloyl chloride in the presence of triethylamine at $0-5^{\circ} \mathrm{C}$ (Scheme 2). The $1 \mathrm{H}-\mathrm{NMR}$ (Figure 2) spectrum of M1 showed characteristic peaks at 5.8 and $6.5 \mathrm{ppm}$ due to vinyl and methyl protons. The aliphatic ethoxy protons showed signals between 1.3 and $4.3 \mathrm{ppm}$. The 


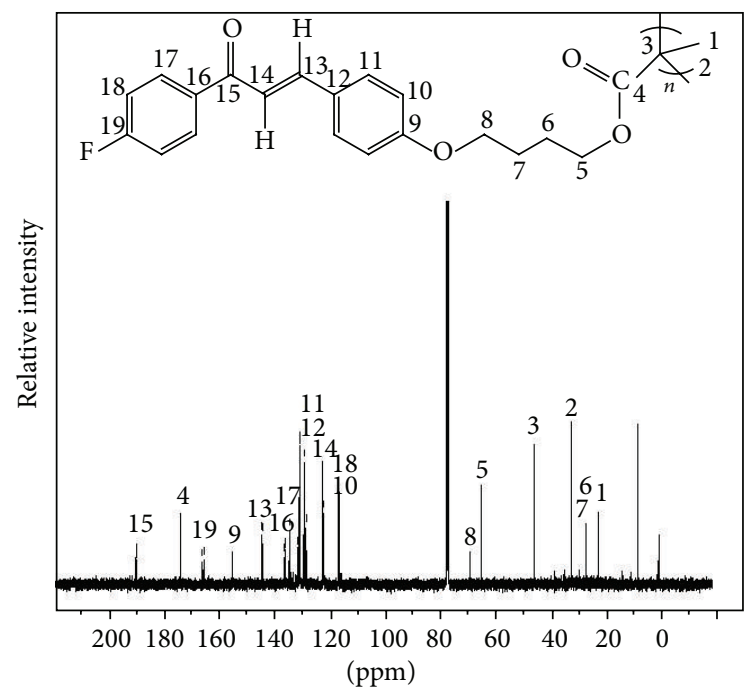

Figure $4:{ }^{13} \mathrm{C}-\mathrm{NMR}$ spectrum of poly(4-[ $4^{\prime}$-flurobenzoylstyryloxy]butyl methacrylate) (FBSOBMA) (P1).

pendent olefinic double bond was shown by signals at 7.5 and $7.9 \mathrm{ppm}$. The appearance of multiplet signals between 7.0 and $8.3 \mathrm{ppm}$ is due to aromatic protons. The above same trends were exhibited by M2 (Figure 3) with slight deviations. The photoresponsive liquid crystalline polymers $\mathrm{P} 1$ and $\mathrm{P} 2$ were synthesized by predetermined amount of monomers in THF at $70^{\circ} \mathrm{C}$; the polymerization was carried out for 48 hours (Scheme 2). The above mixture was precipitated in methanol and filtered off. The precipitated polymer was separated by filtration and purified by repeated reprecipitation from chloroform into methanol. The polymers synthesized were identified using ${ }^{13} \mathrm{C}$-NMR spectra. The ${ }^{13} \mathrm{C}$-NMR of P1 is shown in Figure 4. The methyl, vinyl, and tertiary carbons resonate between 23.5 and $70 \mathrm{ppm}$. The ester and olefinic carbons were identified by the signals at $174.6,122$, and $144.5 \mathrm{ppm}$, respectively. The signals between 118 and $167 \mathrm{ppm}$ were due to aromatic double-bonded carbons. ${ }^{13} \mathrm{C}-\mathrm{NMR}$ spectra polymer P2 is shown in Figure 5. The methyl, vinyl, and tertiary carbons resonate $25.5-69 \mathrm{ppm}$. The ester and olefinic carbons were identified by the signals at 175.5, 121.5, and $143.5 \mathrm{ppm}$ respectively. The signals $116-170 \mathrm{ppm}$ were due to aromatic double-bonded carbons. The solubility of both the polymers P1 and P2 was tested in various organic solvents. They were soluble in polar aprotic solvents such as DMF, DMSO, Dioxane, and THF and in chlorinated solvents such as chloroform, dichloromethane. It was insoluble in methanol, 2-propanol, and hydrocarbon solvents such as toluene, benzene and $n$-hexane.

4.2. Molecular Weight. The number average and weight average molecular weight of polymers $\mathrm{P} 1$ and $\mathrm{P} 2$ were determined by PL-GPC650. The number average molecular weight $\left(\overline{M_{n}}\right)$ and weight average molecular weight $\left(\overline{M_{w}}\right)$ of the polymer P1 were $8.7 \times 10^{4}$ and $13.05 \times 10^{4}$. The molar mass distribution of polymer is given by polydispersity index (PDI) value 1.50 . For the polymer P2, the $\overline{M_{n}}$ and $\overline{M_{w}}$ were $8.92 \times 10^{4}$ and

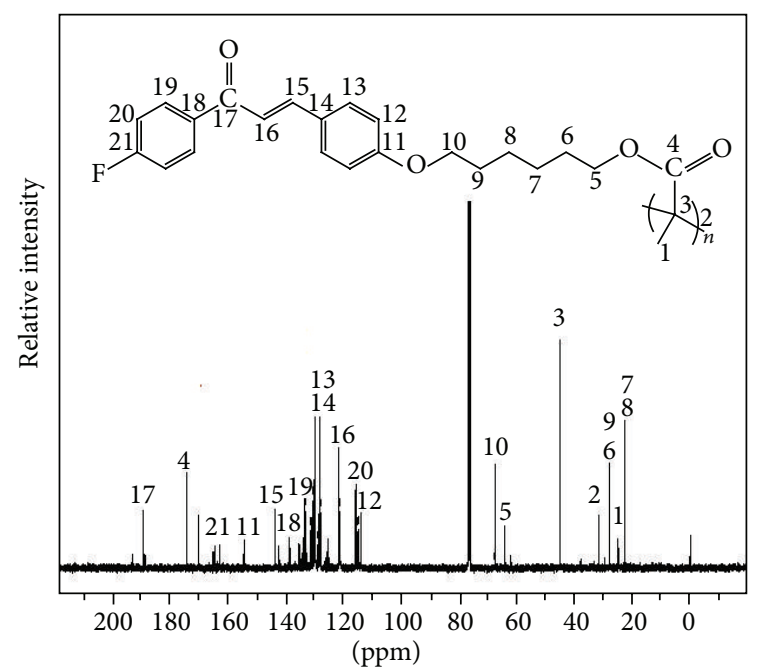

FIGURE 5: ${ }^{13} \mathrm{C}$-NMR spectrum of poly(6-[ $44^{\prime}$-flurobenzoylstyryloxy]hexyl methacrylate) (FBSOHMA) (P2).

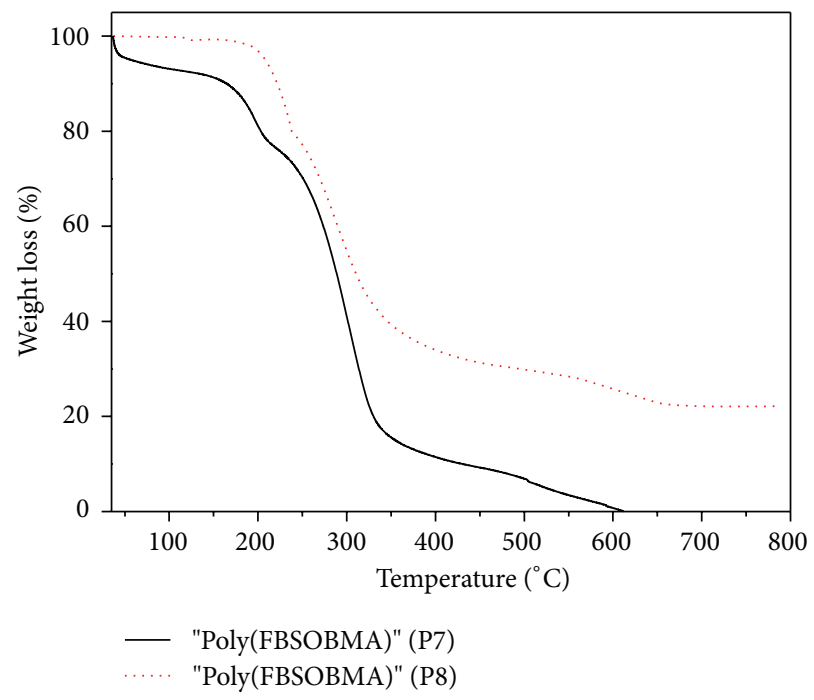

Figure 6: Thermogravimetric Analysis of poly(4-[4'flurobenzoylstyryloxy] butyl methacrylate) (P1) and poly(6[4'-flurobenzoylstyryloxy] hexyl methacrylate) (P2).

$13.46 \times 10^{4}$, respectively, and polydispersity index $(\mathrm{PDI})$ value was 1.51 . The theoretical values of PDI for polymer produced via radical combination and disproportionation are 1.5 and 2.0 , respectively. The PDI values of polymers $\mathrm{P} 1$ and $\mathrm{P} 2$ were 1.50 and 1.51, respectively. Both polymers $\mathrm{P} 1$ and $\mathrm{P} 2$ show the tendency of chain termination by radical combination rather than disproportionation.

4.3. Thermal Properties. The Thermogravimetric Analysis (TGA) of prepared polymers was measured under nitrogen atmosphere in the temperature ranges $30-700^{\circ} \mathrm{C}$ in order to investigate the thermal stability. The TGA data are illustrated in Table 1. The data in Table 1 and Figure 6 indicate that the homopolymers decompose at higher temperature, and they 
TABLE 1: Thermogravimetric Analysis (TGA) and liquid crystalline properties data of polymers (P1 and P2).

\begin{tabular}{lcccccccc}
\hline S. no. & \multirow{2}{*}{ Polymer } & $\begin{array}{c}\text { Temperature }\left({ }^{\circ} \mathrm{C}\right) \text { at weight loss }(\%) \\
\text { IDT }\left({ }^{\circ} \mathrm{C}\right)\end{array}$ & $50 \%$ & $T_{g}\left({ }^{\circ} \mathrm{C}\right)$ & $T_{m}\left({ }^{\circ} \mathrm{C}\right)$ & $T_{i}\left({ }^{\circ} \mathrm{C}\right)$ & $\Delta T=T_{i}-T_{M}\left({ }^{\circ} \mathrm{C}\right)$ & Mesophase \\
\hline P1 & Poly(FBSOBMA) & 160 & 287 & Nil & 105 & 147 & 42 & Nematic \\
P2 & Poly(FBSOHMA) & 194 & 310 & Nil & 97 & 126 & 29 & Nematic \\
\hline
\end{tabular}

show single-stage decomposition with good thermal stability. The initial decomposition temperature (IDT) of the polymers P1 and P2 were slightly different from each other due to smaller difference in molecular mass. Both P1 and P2 show $50 \%$ weight loss at temperatures 287 and $310^{\circ} \mathrm{C}$, respectively, proving the flame retardancy and stability of polymers. These thermal studies have shown that the polymers possess very good thermal and thermooxidative stability required for the negative photoresist polymers.

4.4. Photo-Cross-Linking Studies. The photo-cross-linking studies were carried out to study the changes which occurred in the polymer during UV irradiation to confirm photoresist nature of polymer. The polymer solution was prepared in the concentration range of $10-20 \mathrm{mg} / \mathrm{L}$ using chloroform. It was irradiated with UV-light of $254 \mathrm{~nm}$; the photo-cross-linking ability of the polymer was followed by the rate of disappearance of the $\mathrm{C}=\mathrm{C}$ bond of photosensitive group in the UV spectrum. When the polymers irradiated with UV light of $254 \mathrm{~nm}$, they undergo $2 \pi+2 \pi$ cycloaddition and form photodimers as shown in Scheme 3. The absorption intensity decreases rapidly with increasing irradiation time and band disappears almost completely within three minutes of irradiation. The decrease in the UV absorption intensity due to the cross-linking of polymer through $2 \pi+2 \pi$ cyclodimerisation of $\mathrm{CH}=\mathrm{CH}$ - group of 4-flurobenzoylstyryloxy group leads to formation of cyclobutane ring. The rate of disappearance of double bond in photosensitive group was calculated by the expression [13]:

$$
\text { Rate of conversion }(\%)=\frac{\left(A_{o}-A_{t}\right)}{\left(A_{o}\right)} \times 100 \text {, }
$$

where $A_{o}$ and $A_{t}$ are absorption intensities due to the $>\mathrm{C}=\mathrm{C}<$ group after the irradiation time $t=0$ and $t=T$ respectively.

The UV spectral changes during photo-cross-linking and photoconversions of polymers are shown in Figures 7 and 8. In the polymers P1 and P2, the pendant chalcone unit and polymeric backbone are linked by flexible methylene spacer units. The photo-cross linking rate of P2 was slightly faster than P1. This rapid cross-linking may be attributed to the spacer unit between the photosensitive group and polymer backbone which provide more flexibility and free movement for the side chain which accelerate the increased rate of cross linking [14].

The photolysis studies of various ethylene spacer containing polymers imparted that the rate of photo-cross-linking depends on the length of the methylene chain so the polymer P7 and P8 follow this trend

$$
\text { Hexamethylene }>\text { Butamethylene. }
$$

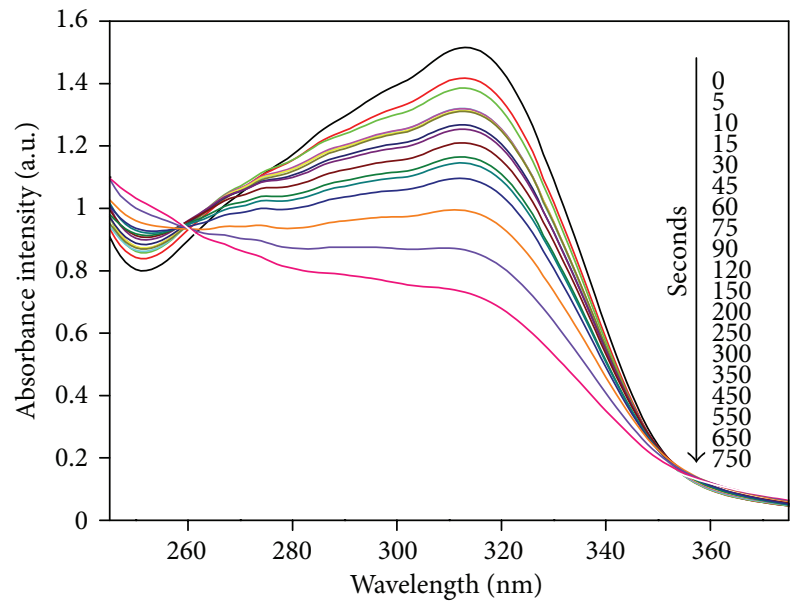

FIGURE 7: UV spectral changes during photo-cross-linking of poly (4-[4'-flurobenzoylstyryloxy]butyl methacrylate) (P7) at various time intervals in chloroform solution.

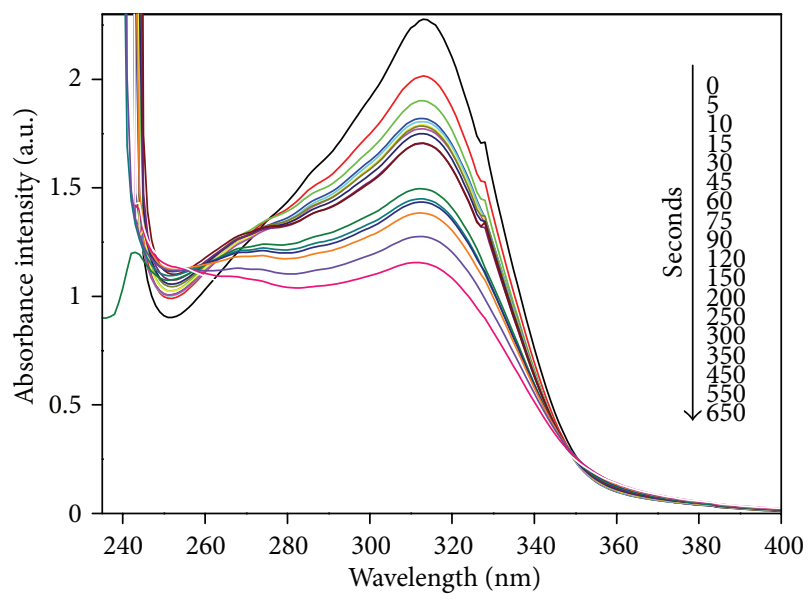

FIGURE 8: UV spectral changes during photo-cross-linking of poly(6-[4'-flurobenzoylstyryloxy]hexyl methacrylate) (P8) at various time intervals in chloroform solution.

Figure 9 denotes that the rate of disappearance of the $\mathrm{C}=\mathrm{C}$ of photoreactive groups is slightly fast in P2 than P1 and shows $100 \%$ photoconversion, since they have smaller substituents in their pendant unit and there is no intramolecular crosslinking can be formed by the dimerization of adjacent chalcone groups.

4.5. Fluorescence Spectral Studies. The existence of photoresponsive behaviour of polymers can be evidenced by fluorescence spectra. The fluorescence intensity of polymer 

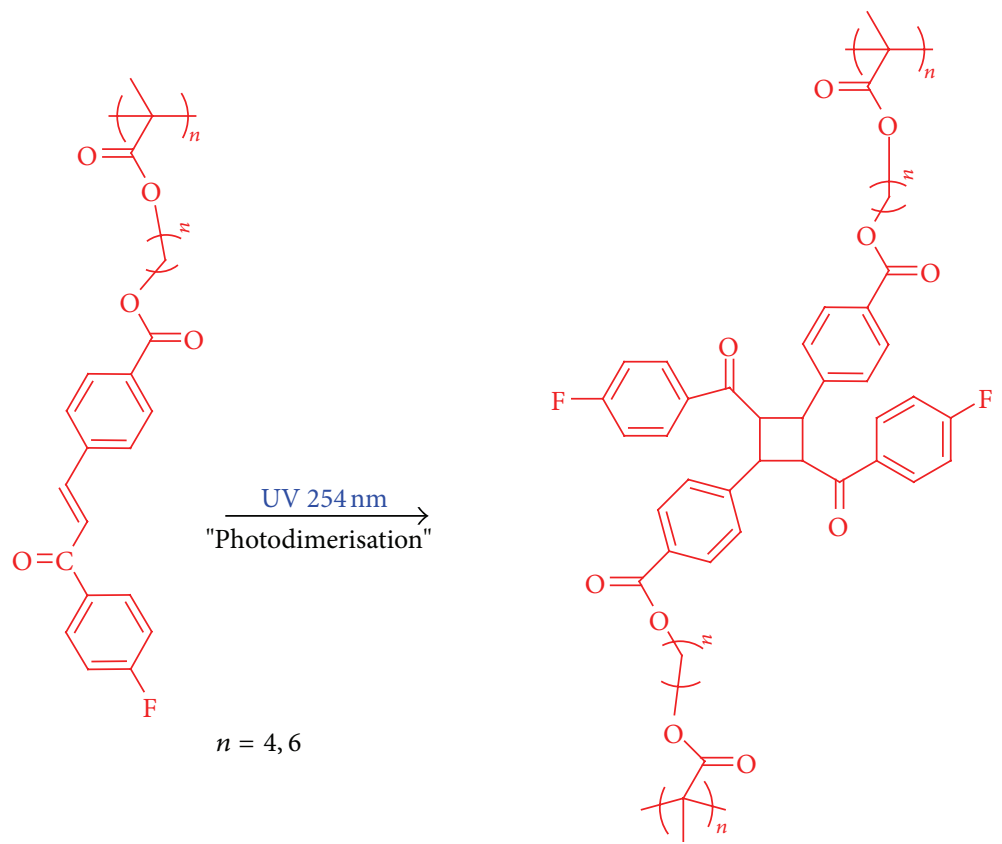

Scheme 3: Photodimerisation of polymers (P1 and P2).

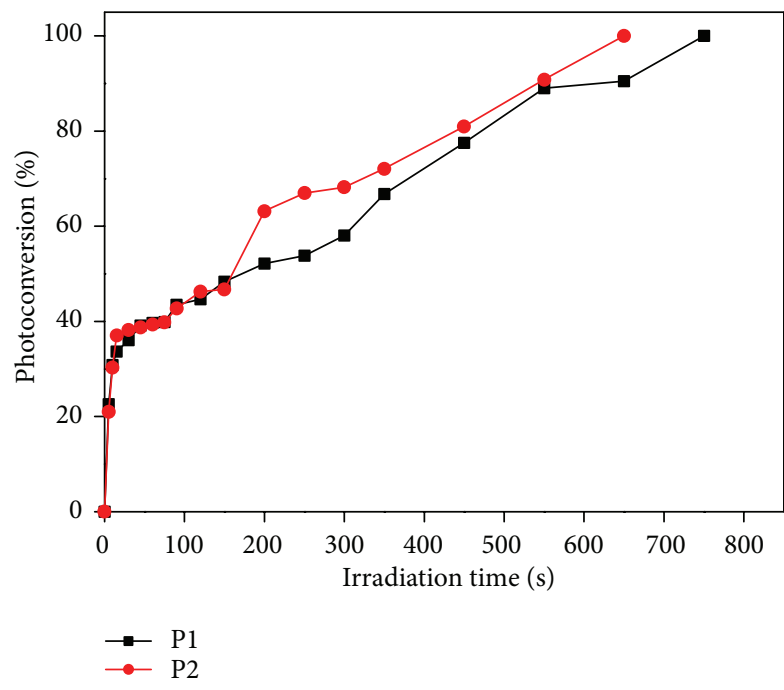

FIgURE 9: Photoconversions on UV-irradiation for polymers P1 and P2.

decreases as the time of irradiation increases. The decrease in intensity is due to $2 \pi+2 \pi$ cycloaddition which leads to cyclobutane ring formation by destroying $\pi$ electron conjugation. From the figures it is noticed that P1 shows gradual decrease in the intensity, whereas $\mathrm{P} 2$ shows sudden decrease.

Fluorescence spectral changes during photo-crosslinking of polymers P1 and P2 are shown in Figures 10 and 11. Both polymers P1 and P2 were excited at the wavelength of $348 \mathrm{~nm}$, and they were irradiated with UV-light of $254 \mathrm{~nm}$ at various time intervals. The decrease in fluorescence intensity

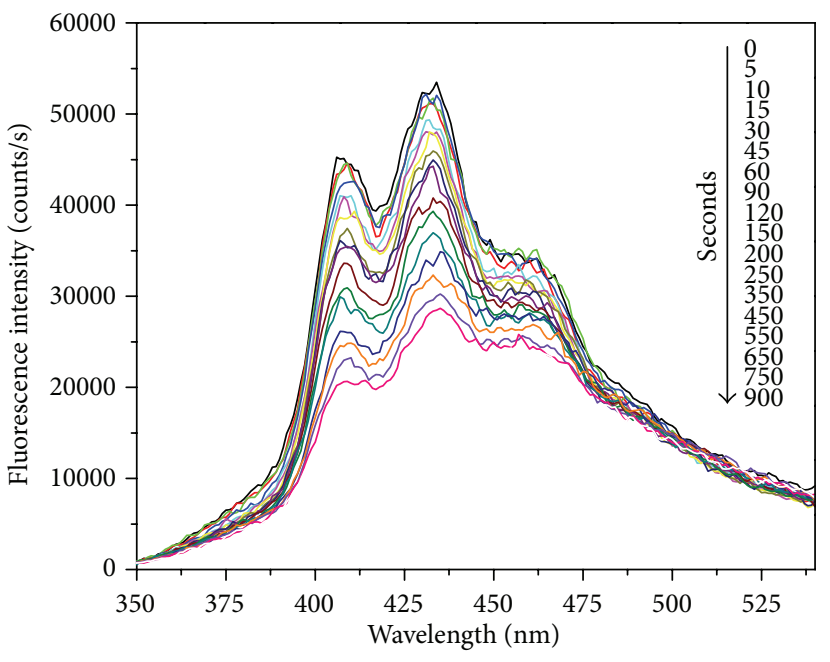

FIGURE 10: Fluorescence spectral changes during photo-crosslinking of poly(4-[4'-flurobenzoylstyryloxy] butyl methacrylate) (P1) at various time intervals in chloroform solution.

was observed till the completion of photo-cross-linking of polymers which is noticed from their decrease in intensity or disappearance of emission peaks. The emission of both polymers P7 and P8 occurred at $430 \mathrm{~nm}$, since they contain similar functional groups and spacer units.

4.6. Morphological Study of Photo-Cross-Linking Polymers. The SEM technique can give high resolution images which enables the visualization of morphological information without losing any accuracy during analysis. The synthesized 


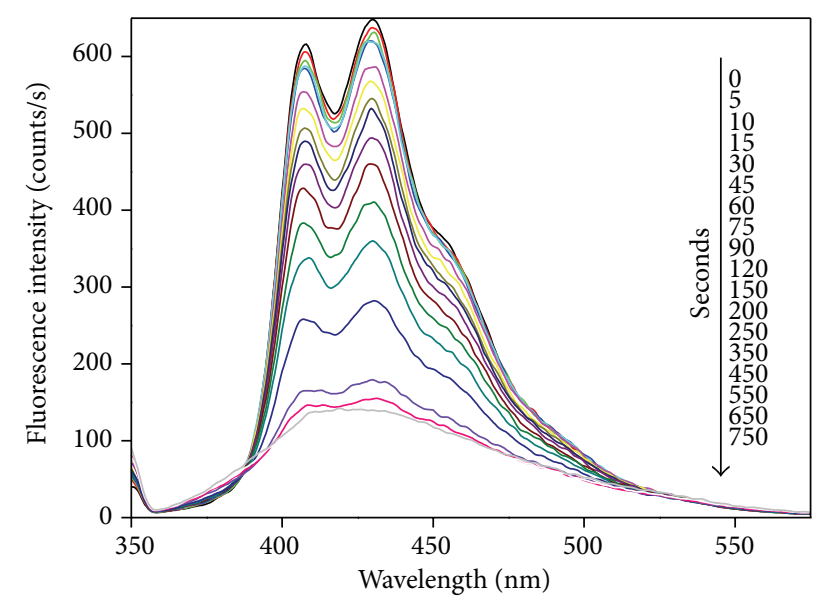

FIGURE 11: Fluorescence spectral changes during photo-crosslinking of poly(6-[ $4^{\prime}$-flurobenzoylstyryloxy]hexyl methacrylate) (P2) at various time intervals in chloroform solution.

photo-cross-linkable liquid crystalline polymers were irradiated with UV-light of $254 \mathrm{~nm}$ for 30 minutes. The virgin polymers (P1 and P2) and photo-cross-linked polymers (P1 and P8) were characterised by HITACHI Scanning Electron Microscope (SEM) S-3400N model to understand the morphology of both virgin and photo-cross-linked polymers. The SEM images of both virgin and photo-cross-linked polymers shown in Figures 12(a) and 13(b). As observed from the SEM images of Figures 12(b) and 13(b), the photo-crosslinked polymer sample confirms loosely held dispersion of polymeric materials, while virgin polymer exhibits compact stringent dispersion of polymeric surface as described by Mathur and Kumar [15].

It is inferred from the SEM images that all the virgin polymers P1-P8 have irregular-shaped flakes in their lattice which packed on one over the other in nondirectional manner, and they all have hard and crystal-like surface. But, SEM images of photo-cross-linked polymers from P1 to P8 show uniform size of polymer flakes which were arranged regularly.

It can be clearly observed from the SEM images that their surfaces after photo-cross-linking were smoothed well, and all the irregular crystal with rough surface has been changed into brightened smooth surface. The smoothness in the polymer surface may be due to photodimerisation of polymers. When two polymer molecules undergo cyclobutane ring formation during photo-cross-linking, one polymer molecule bounds to the other through cyclobutane ring. This structural interaction may lead to smoothness and regular or ordered arrangement of polymer lattice after UV-treatment.

4.7. Liquid Crystalline Properties of Polymers. The development of photosensitive media based on liquid crystalline compounds for data recording, optical storage, and reproduction is one of the most rapidly developing areas in the physical chemistry of low molecular mass and polymer liquid crystals [16]. The rigidity of the mesogenic core, the flexible spacer length, and terminal units highly influence the molting

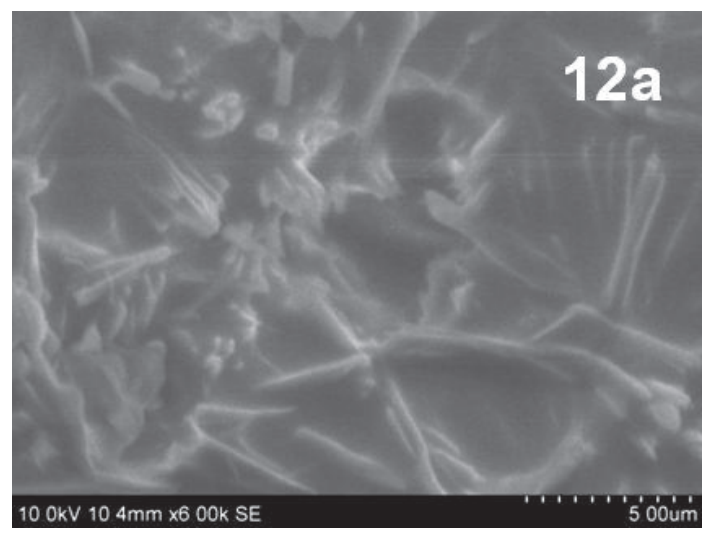

(a)

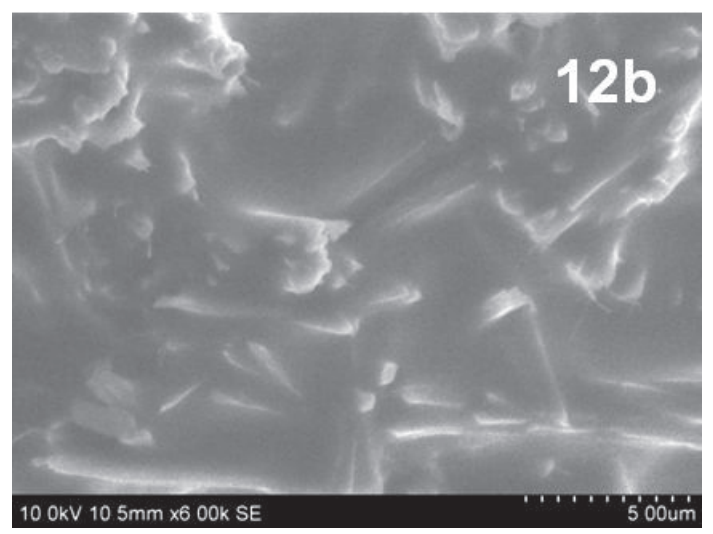

(b)

FIgURE 12: Scanning Electron Microscope image of (a) virgin polymer poly(FBSOBMA) (P1) and (b) photo-cross-linked polymer poly(FBSOBMA) (P1).

temperature, mesophase temperature, and even molecular arrangement.

In some polymers, they are taking the effect of mesogen and spacer together; a polymer having rigid mesogen and shorter spacer should show the higher transition temperature [17]. The phase transition temperature and mesophase of the polymers are studied by traces of DSC thermogram and HPOM images. Generally in the DSC thermogram, at the highest transition temperature there will be an endotherm corresponding to the transition from LC phase to isotropic phase. The transition in some cases from crystal to liquid crystal is marked by more than one endotherm. When such multiple curves were observed, the one having the highest temperature is attributed to crystal-to-mesophase transition.

The DSC thermograms of Polymers $\mathrm{P} 1$ and $\mathrm{P} 2$ are shown in the Figure 14 which shows two endotherms observed in the heating scan and in the annealed samples. In general, for the first lowest transition temperature which occur just after the glass transition temperature is melting endotherm, and the second highest transition temperature attributed to nematic phase to isotropic mesophase transition. The polymers $\mathrm{P} 1$ and $\mathrm{P} 2$ show crystal-to-nematic phase transition at temperatures at $105^{\circ} \mathrm{C}$ and $97^{\circ} \mathrm{C}$, respectively. The polymers 


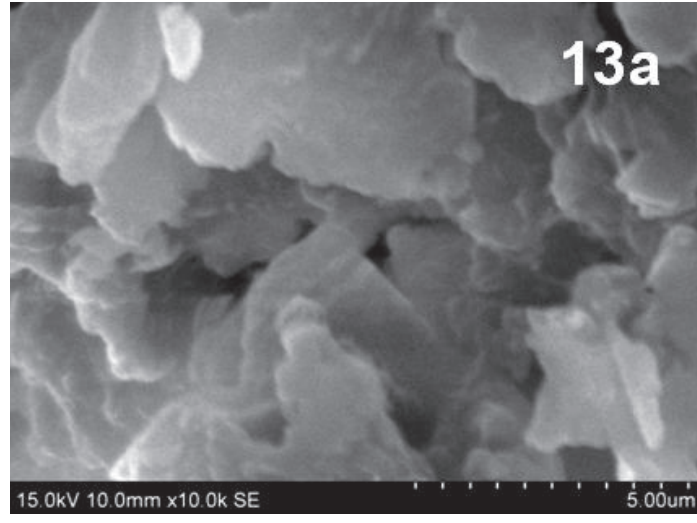

(a)

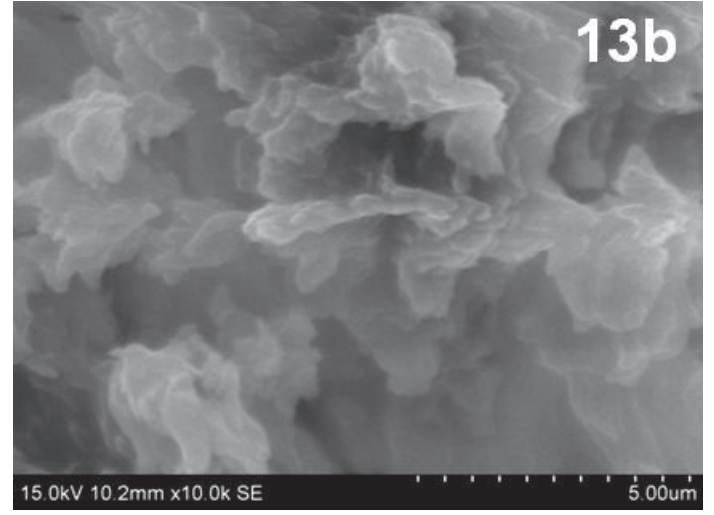

(b)

Figure 13: Scanning Electron Microscope image of (a) virgin polymer poly (FBSOHMA) (P2) and (b) photo-cross-linked polymer poly (FBSOHMA) (P2).

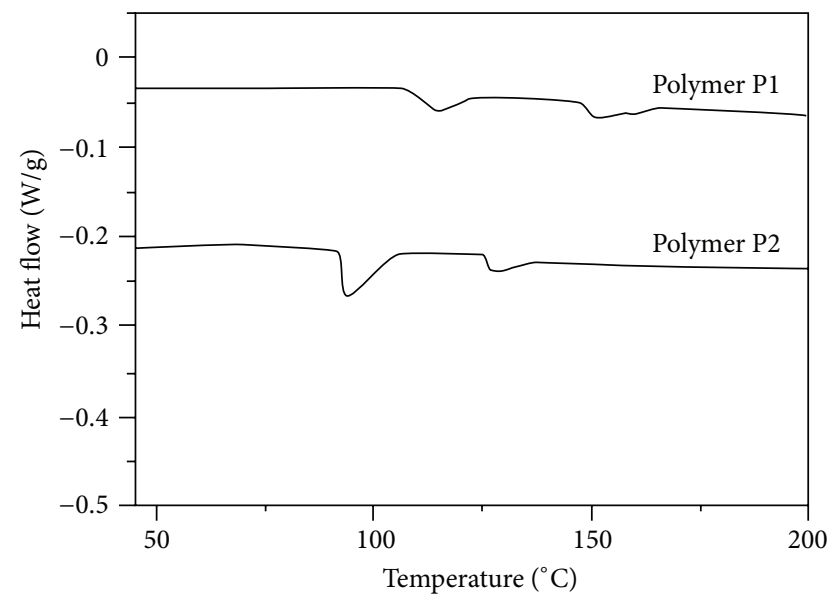

FIGURE 14: Differential scanning calorimetric thermogram of polymers poly(FBSOBMA) (P1) and poly(FBSOHMA) (P2).

$\mathrm{P} 1$ and $\mathrm{P} 2$ show nematic-to-isotropic phase transition at the temperature $147^{\circ} \mathrm{C}$ and $126^{\circ} \mathrm{C}$, respectively shown in Figures 15 and 16. The liquid crystalline properties of polymer are shown in Table 1 . The liquid crystalline phases were observed in between the $T_{m}$ and $T_{i}$. The mesophase duration $(\Delta T=$ $T_{i}-T_{m}$ ) was observed in between 42 and $29^{\circ} \mathrm{C}$; this indicates that they possess relatively good mesophase stability.

4.8. Fluorescence Lifetime Studies. The time-resolved fluorescence decays of polymers P1 and P2 have been monitored in chloroform. The polymers P1 and P2 were excited at $460 \mathrm{~nm}$. The fluorescence lifetimes of both polymers after and before the UV-irradiation at wavelength of $254 \mathrm{~nm}$ have been measured and plotted in Figures 17(a) and 18(c). The $\chi^{2}$ values which are known as the fitting parameters determining the fine fit for triexponential decay are found to be $\langle 1.3$, and average lifetime $\langle\tau\rangle$ is calculated using the following equation [18]:

$$
\tau_{\mathrm{av}}=\frac{\alpha_{1} \tau_{1}^{2}+\alpha_{2} \tau_{2}^{2}+\alpha_{3} \tau_{3}^{2}}{\alpha_{1} \tau_{1}+\alpha_{2} \tau_{2}+\alpha_{3} \tau_{3}}
$$

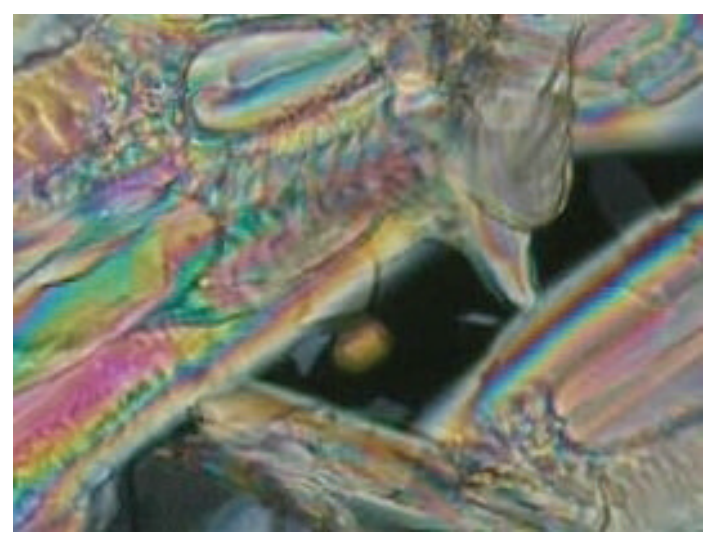

FIGURE 15: Polarised optical micrograph of poly(FBSOBMA) (P1) showing nematic mesophase at $147^{\circ} \mathrm{C}$.

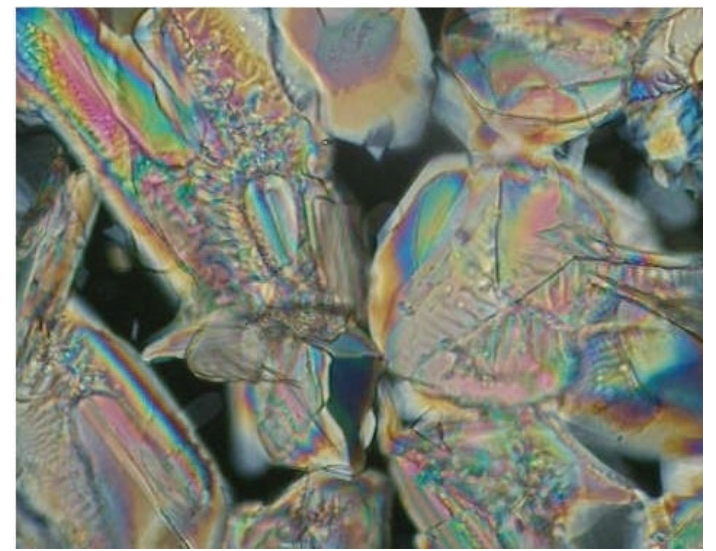

FIGURE 16: Polarised optical micrograph of poly(FBSOHMA) (P2) showing nematic mesophase at $126^{\circ} \mathrm{C}$.

where $\tau_{1}, \tau_{2}$, and $\tau_{3}$ are the lifetime values of three emissive states and $\alpha_{1}, \alpha_{2}$, and $\alpha_{3}$ are called preexponential factors which give abundance of emissive states. The fluorescence lifetime values of photoresponsive polymers P1 and P2 were 


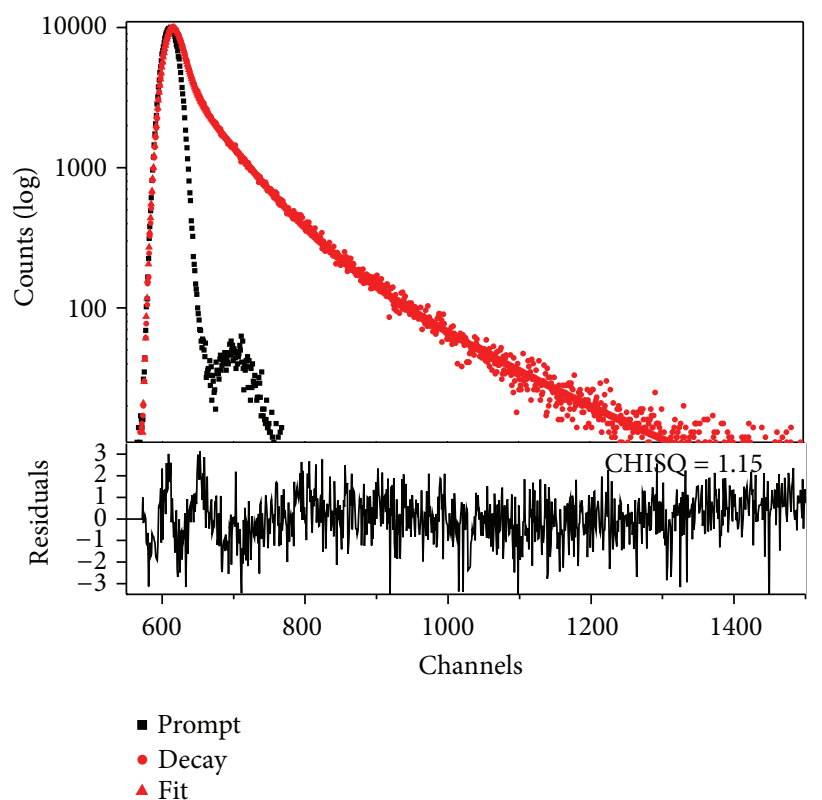

(a)

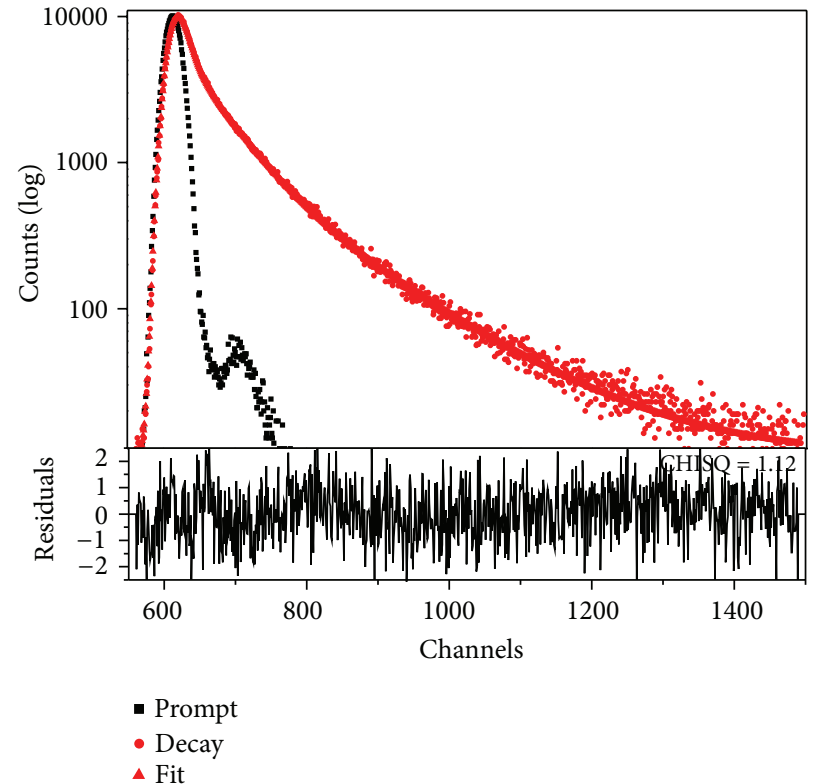

(b)

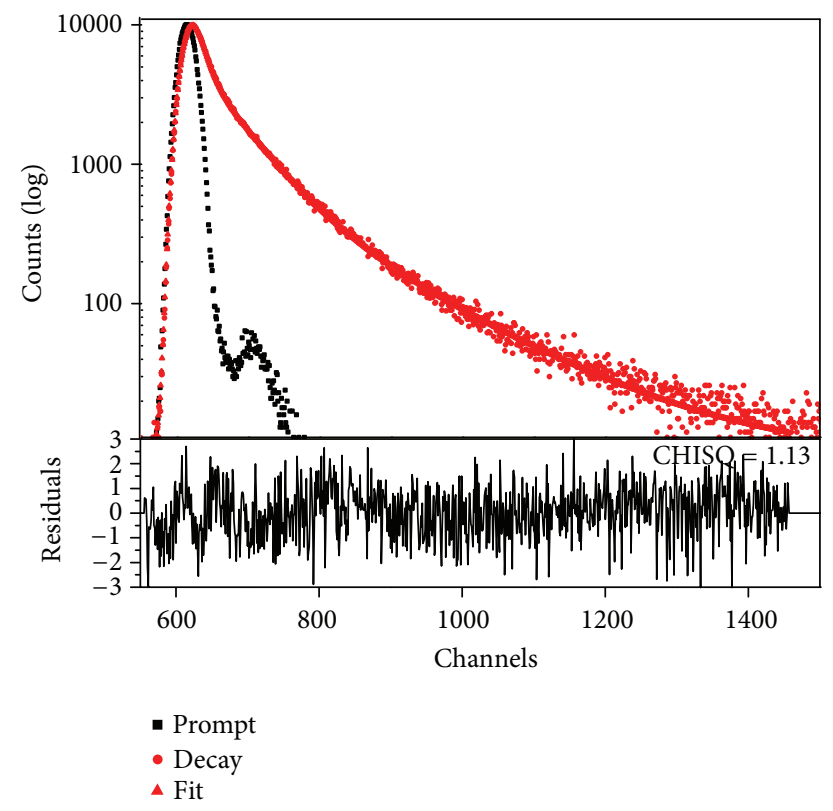

(c)

FIgURE 17: (a) Lifetime decay of poly(4-[4'-flurobenzoylstyryloxy]butyl methacrylate) (P1) before UV-irradiation. (b) Lifetime

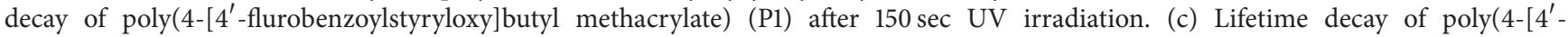
flurobenzoylstyryloxy] butyl methacrylate) (P1) after infinite sec UV irradiation.

given in Table 2 . The polymer solutions were prepared in the concentration range $10-20 \mathrm{mg} / \mathrm{L}$ using chloroform. The lifetime measurements of two polymers were determined at nonirradiative and UV-irradiative condition at different time intervals. Since the number of molecules with electron donor or withdrawing groups affects fluorescence only partially [19], the polymers show very little lifetime variations before and after UV-irradiations. In both polymers P1 and P2, lifetime values are decreasing after UV-irradiation at different time intervals 0,150 , and $\infty$ seconds.

In general, rotation of the part of the molecule participating in fluorescence is the most trivial process of the nonirradiative energy loss and typically occurs in the excited state. Considering the molecules of the bond order in ground state equal to 2 , upon excitation the electron from the bonding orbital is promoted to the excited state orbital 

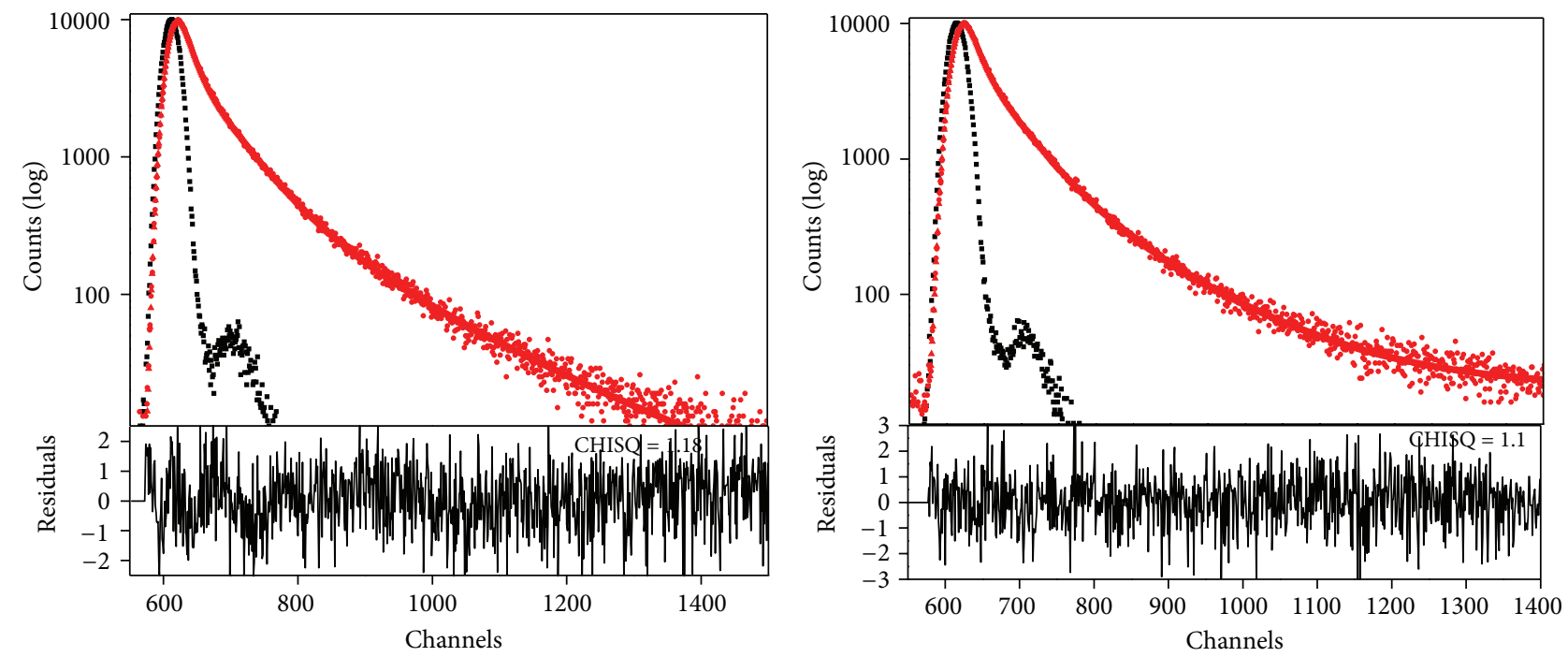

- Prompt
- Decay
- Fit

- Prompt

- Decay

$\Delta$ Fit

(a)

(b)

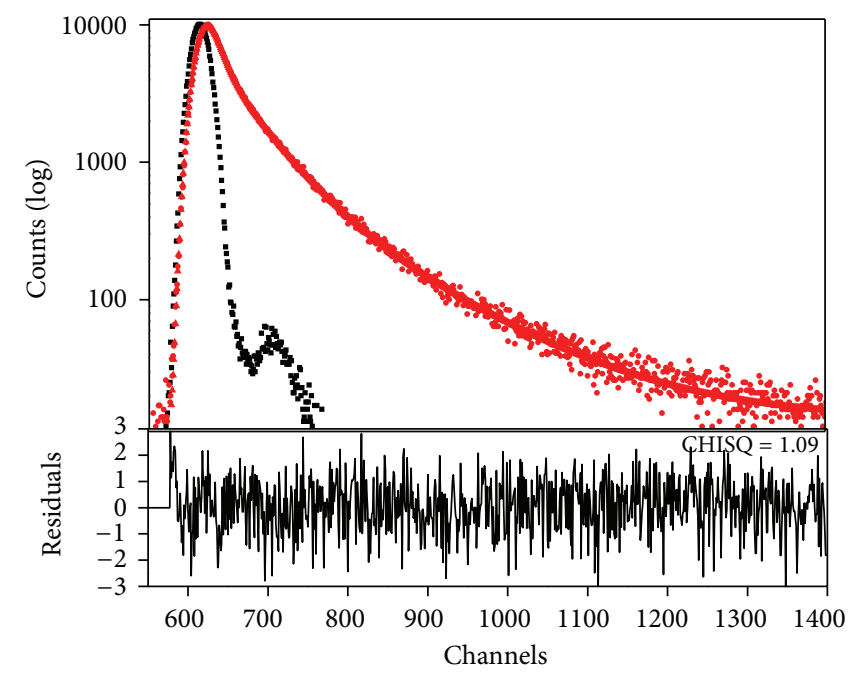

- Prompt

- Decay

$\Delta$ Fit

(c)

Figure 18: (a) Lifetime decay of poly(6-[4'-flurobenzoylstyryloxy]hexyl methacrylate) (P2) before UV-irradiation. (b) Lifetime decay of poly(6-[4'-flurobenzoylstyryloxy]hexyl methacrylate) (P2) after $150 \mathrm{sec}$ UV irradiation. (c) Lifetime decay of poly(6-[4'flurobenzoylstyryloxy] hexyl methacrylate) (P2) after infinite sec UV irradiation.

TABLE 2: Fluorescence lifetime decay of polymers P1 and P2.

\begin{tabular}{|c|c|c|c|c|c|c|c|c|c|c|c|}
\hline Polymers & $\lambda_{A}(\mathrm{~nm})$ & $\lambda_{E}(\mathrm{~nm})$ & UV-irradiation time (sec) & $\tau_{1}(\mathrm{~ns})$ & $\alpha_{1}$ & $\tau_{2}(\mathrm{~ns})$ & $\alpha_{2}$ & $\tau_{3}(\mathrm{~ns})$ & $\alpha_{3}$ & $\langle\tau\rangle$ & $\chi^{2}$ \\
\hline \multirow{3}{*}{ P1 } & \multirow{3}{*}{313} & \multirow{3}{*}{430} & 0 & 3.07 & 0.4601 & 0.4287 & 0.3657 & 9.05 & 0.1742 & 5.94 & 1.15 \\
\hline & & & 150 & 2.72 & 0.4417 & 0.4761 & 0.2899 & 7.63 & 0.2683 & 5.60 & 1.12 \\
\hline & & & $\infty$ & 2.40 & 0.4349 & 0.4469 & 0.2790 & 7.12 & 0.2862 & 5.32 & 1.06 \\
\hline \multirow{3}{*}{ P2 } & \multirow{3}{*}{313} & \multirow{3}{*}{430} & 0 & 3.19 & 0.4946 & 0.8282 & 0.3312 & 9.60 & 0.1742 & 6.05 & 1.18 \\
\hline & & & 150 & 2.65 & 0.5065 & 0.6778 & 0.2663 & 7.23 & 0.2271 & 4.91 & 1.10 \\
\hline & & & $\infty$ & 2.61 & 0.4848 & 0.6154 & 0.2926 & 7.01 & 0.2226 & 4.77 & 1.09 \\
\hline
\end{tabular}


producing bond order 1 . Such change in the bond order transforms the rigid frame work formed by the double bond to a flexible system of single bond, leading to twisting of molecule around a C-C bond causing subsequent cis/trans isomerisation [20]. Since rotation around the double bonds contributes to a decrease in lifetime, it is logical to suggest that any restriction of rotation, such as rigid environment of the molecules, would marginalize the role of nonradiative path way and lead to subsequent increase of fluorescence lifetime.

Molecules capable of undergoing an electron transfer process possess strong electron donating and occasionally electron withdrawing group [21]. Among withdrawing groups, only nitro group has been used successfully as quenchers other withdrawing groups may affect a little fluorescence lifetime. For example, tetranitrofluorescence has lifetime of $2.4 \mathrm{~ns}$ compared with $4.0 \mathrm{~ns}$ for fluorescence.

The polymers poly $\left(4-\left[4^{\prime}\right.\right.$-flurobenzoylstyryloxy $]$ butyl methacrylate) (P1) and poly(6-[4'-flurobenzoylstyryloxy] hexyl methacrylate) (P2) fluorescence lifetime values before irradiation were 5.94 and 6.05. The molecules capable of undergoing an electron transfer possess strong electron donating and occasionally electron withdrawing group. The exceptions are however numerous, and a number of molecules with electron donor or electron withdrawing group affect fluorescence lifetime partially. So the electronegative nature of flurogroup substitution at the 4 th position of both polymers $\mathrm{P} 1$ and $\mathrm{P} 2$ might not be affected by the fluorescence lifetime values. Nevertheless, the two polymers showed restriction to free rotation after photodimerisation, and slight polarisation of ester linkage predominates into further decrease of fluorescence lifetime up to infinite photodimerisation. In the polymers P1 and P2 they show $\chi^{2}$ values near to 1.1 or 1 which indicate all the fits are monoexponential and show the goodness of fitting parameters.

\section{Conclusions}

The photos-cross-linkable, liquid crystalline polymers $\mathrm{P} 1$ and P2 were synthesized by free radical polymerization in THF using AIBN as initiator. The synthesized polymers have been characterized by $\mathrm{H}^{1}$-NMR, $\mathrm{C}^{13}$-NMR, and UV-Vis spectral studies. The TGA analysis clearly indicates that the polymers show $50 \%$ weight loss near to $400^{\circ} \mathrm{C}$ which exhibit good characteristics of thermal and thermo-oxidative stability. The polydispersity index (PDI) values 1.50 and 1.51 obtained from GPC indicate that polymerization was terminated by free radical combination. The photo-cross-linking and fluorescence lifetime study of polymers show their indispensable importance in photoresist applications. The liquid crystalline property of the polymers was identified from DSC and confirmed by HOPM images at $147^{\circ} \mathrm{C}$ and $126^{\circ} \mathrm{C}$. From the average lifetime values $5.32 \mathrm{~ns}$ and $4.77 \mathrm{~ns}$ at infinite UVirradiation on both $\mathrm{P} 1$ and $\mathrm{P} 2$ reveal that the photo physical behavior of polymers using the time-correlated single photon counting (TCSPC) method. Thus, the synthesized polymers exhibit both photoresponsive as well as liquid crystalline property, and they might be useful in optical data recording and nonlinear optical (NLO) applications.

\section{Acknowledgments}

The authors are thankful to Professor (Dr.) S. Ganesan, Director of Students Affair, Anna University, Chennai, India, for providing fluorescence lifetime facility to carry out this research and sincerely thankful to Dr. T. Narasimhaswamy, Scientist, CLRI, Adyar, Chennai, for carrying out POM studies.

\section{References}

[1] D. Creed, A. C. Griffin, J. R. D. Gross, C. E. Hoyle, and K. Venkataram, "Molecular crystals and liquid crystals incorporating nonlinear optics," Molecular Crystals and Liquid Crystals, vol. 155, no. 1, pp. 57-71, 1988.

[2] T. Ikeda, H. Itakura, C. Lee, F. M. Winnik, and S. Tazuke, "Topochemical photodimerization in polymer liquid crystals," Macromolecules, vol. 21, no. 12, pp. 3536-3537, 1988.

[3] P. Keller, "Photo-crosslinkable liquid-crystalline side-chain polysiloxanes," Chemistry of Materials, vol. 2, no. 1, pp. 3-4, 1990.

[4] M. J. Whitecombe, A. Gilbert, A. Hiraj, and G. R. Mitchell, "Cinnamate ester containing liquid crystalline side chain polymers," Journal of Polymer Science Part A, vol. 29, no. 2, pp. 251-259, 1991.

[5] M. J. Whitecombe, A. Gilbert, and G. R. Mitchell, "The photoFries rearrangement in a side-chain liquid-crystalline polymer," Polymer, vol. 34, no. 7, pp. 1347-1353, 1993.

[6] A. C. Griffin, C. E. Hoyle, J. R. D. Gross, K. Venkataram, D. Creed, and C. B. McArdle, "Laser-induced photo-optical recording on free-standing films of a main-chain nematic polyester," Die Makromolekulare Chemie, vol. 9, no. 7, pp. 463$477,1988$.

[7] C. H. Legge, M. J. Whitcombe, A. Gilbert, and G. R. Mitchell, "Photoinduced phase transitions in novel liquid-crystalline copolymers," Journal of Materials Chemistry, vol. 1, no. 2, pp. 303-304, 1991.

[8] S. Marturukakul, I. J. Chen, L. Li, R. J. Jeng, J. Kumar, and S. K. Tripathy, "An interpenetrating polymer network as a stable second-order nonlinear optical material," Chemistry of Materials, vol. 5, no. 5, pp. 592-594, 1993.

[9] W. Becker, Advanced Time-Correlated Single Photon Counting Techniques, , Springer, Berlin, Germany, 2005.

[10] W. Becker, The TCSPC Handbook, Becker \& Hickl Gmbh, Berlin, Germany, 3rd edition, 2008.

[11] A. V. Rami Reddy, K. Subramanian, V. Krishnasamy, and J. Ravichandran, "Synthesis, characterization and properties of novel polymers containing pendant photocrosslinkable chalcone moiety," European Polymer Journal, vol. 32, no. 8, pp. 919926, 1996.

[12] A. V. Rami Reddy, K. Subramanian, and J. Seshasainath, "Photosensitive polymers: synthesis, characterization, and photocrosslinking properties of polymers with pendant $\alpha, \beta$ unsaturated ketone moiety," Journal of Applied Polymer Science, vol. 70, no. 11, pp. 2111-2120, 1998.

[13] K. Subramanian, V. Krishnasamy, S. Nanjundan, and A. V. Rami Reddy, "Photosensitive polymer: synthesis, characterization and properties of a polymer having pendant photocrosslinkable group," European Polymer Journal, vol. 36, no. 11, pp. 2343-2350, 2000 . 
[14] R. Mohan Kumar, C. Saravanan, S. Senthil, and P. Kannan, "Synthesis, characterization and photolysis studies on liquid crystalline poly[4-(4'-x-biphenyl)yl-4" -(m-methacryloyloxyalkyloxy) cinnamate]'s," European Polymer Journal, vol. 43, no. 6, pp. 2648-2659, 2007.

[15] S. C. Mathur and B. Kumar, "Charge transport calculations in anthraquinone," Molecular Crystals and Liquid Crystals, vol. 23, no. 1-2, pp. 85-98, 1973.

[16] A. Bobrovsky and V. Shibaev, "A study of photooptical processes in photosensitive cholesteric azobenzene-containing polymer mixture under an action of the polarized and nonpolarized light," Polymer, vol. 47, no. 12, pp. 4310-4317, 2006.

[17] Gangadhara and K. Kishore, "A new class of photo-crosslinkable side chain liquid crystalline polymers containing bis(benzylidene)cyclohexanone units," Macromolecules, vol. 28, no. 4, pp. 806-815, 1995.

[18] J. R. Lakowicz, Principles of Fluorescence Spectroscopy, Kluwer Academic/Plenum Publishers, New York, NY, USA, 2nd edition, 1999.

[19] N. O. McHedlov-Petrossyan, N. A. Vodolazkaya, Y. N. Surov, and D. V. Samoylov, "2,4,5,7-tetranitrofluorescein in solutions: novel type of tautomerism in hydroxyxanthene series as detected by various spectral methods," Spectrochimica Acta Part A, vol. 61, no. 11-12, pp. 2747-2760, 2005.

[20] A. J. Merer and R. S. Mulliken, "Ultraviolet spectra and excited states of ethylene and its alkyl derivatives," Chemical Reviews, vol. 69, no. 5, pp. 639-656, 1969.

[21] V. Knyukshto, E. Zenkevich, E. Sagun, A. Shulga, and S. Bachilo, "Pathways for photoinduced electron transfer in mesonitro-phenyl-octaethylporphyrins and their chemical dimers," Chemical Physics Letters, vol. 304, no. 3-4, pp. 155-166, 1999. 

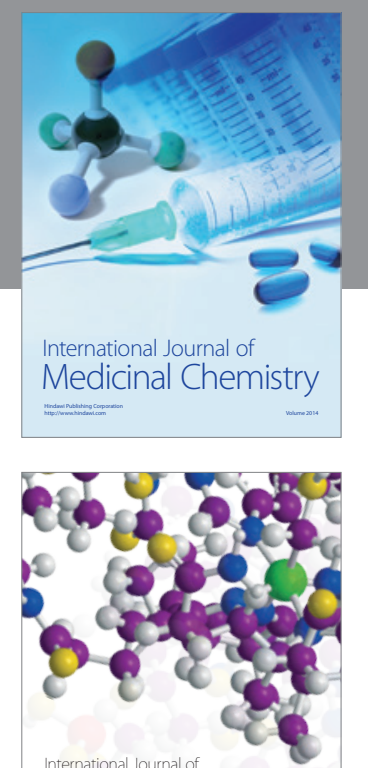

\section{Carbohydrate} Chemistry

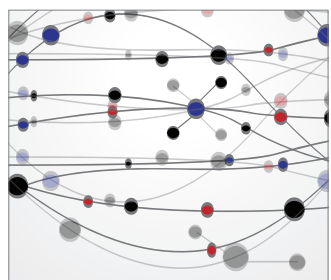

The Scientific World Journal
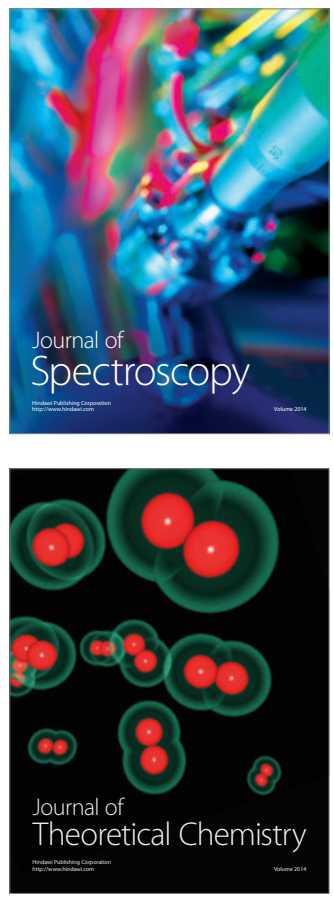
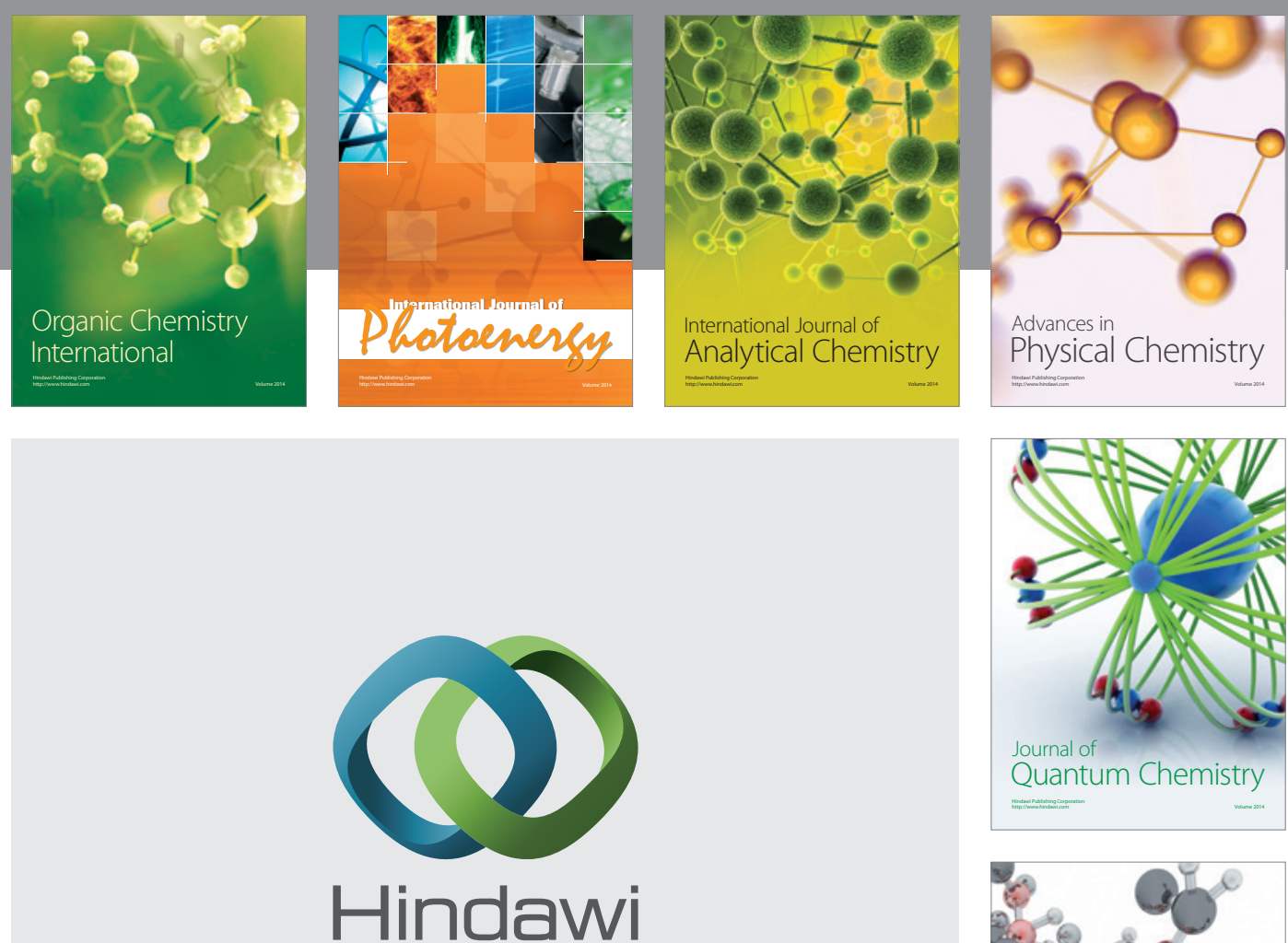

Submit your manuscripts at

http://www.hindawi.com

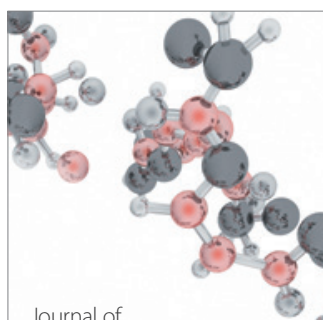

Analytical Methods

in Chemistry

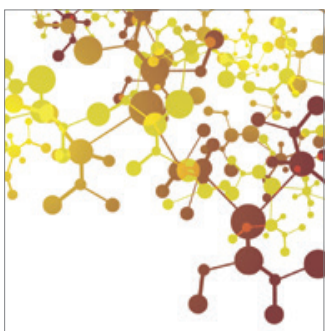

Journal of

Applied Chemistry

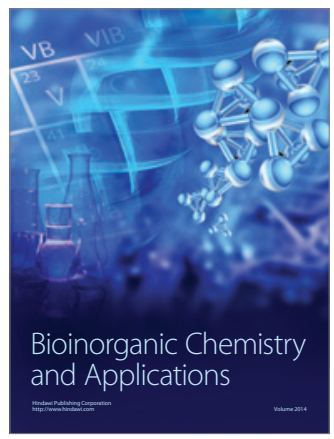

Inorganic Chemistry
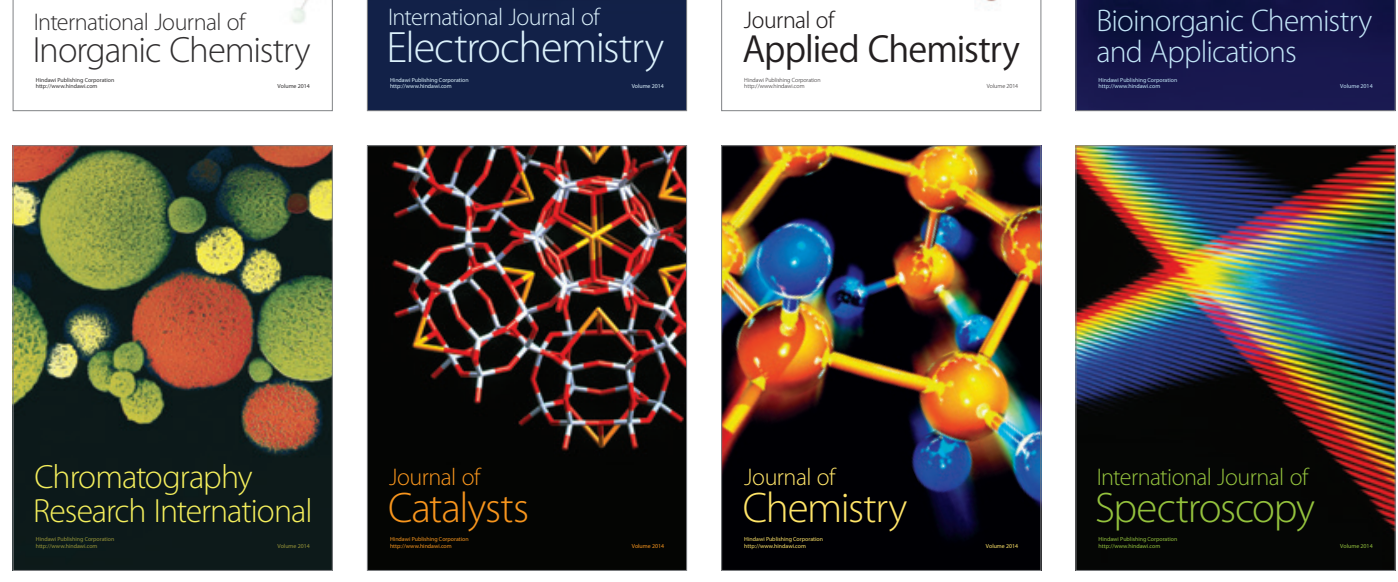